\title{
El Uso de Datos de Evaluaciones Educativas en Brasil, Chile y Argentina
}

\author{
Use of Educational Assessment Data in Brazil, Chile and \\ Argentina
}

\author{
Luís Gustavo Amaral Vinha* y Jacob Arie Laros \\ Universidad de Brasília
}

\begin{abstract}
La disponibilidad de información relacionada con evaluaciones educacionales es grande en América Latina, principalmente después de las reformas educativas de las últimas décadas. El objetivo de este estudio es trazar un mapa de cómo los datos de las evaluaciones educativas realizadas en Brasil, Chile y Argentina son utilizados por los investigadores. La metodología empleada para la identificación y selección de los estudios fue la búsqueda de publicaciones, entre 1994 y 2014, por medio de la base de datos de la agencia CAPES. Utilizando las palabras clave: "rendimiento escolar" (en español, portugués - rendimento escolar - e inglés - school performance), SAEB, SIMCE y "Operativo Nacional de Evaluación"; se seleccionaron 27 estudios empíricos que utilizan datos de las evaluaciones de los tres países en cuestión. La revisión mostró que la técnica más utilizada fue el análisis de regresión multinivel. Se verificó que casi todos los trabajos seleccionados analizaban el impacto de factores asociados al desempeño escolar. Sin embargo, también se utilizaron los datos de las evaluaciones educativas para evaluar el papel de la escuela en la promoción de la equidad y estimar el impacto de programas educativos. Los resultados obtenidos en este estudio permiten concluir que, en general, los artículos revisados proporcionan información que puede apoyar políticas que contribuyan a la mejora de la educación.
\end{abstract}

Palabras clave: Rendimiento escolar, Evaluación educacional, Factores asociados al rendimiento escolar, Efecto escuela, Evaluación de programas sociales, Análisis multinivel, Educación básica, Educación secundaria, América Latina.

\begin{abstract}
A great amount of information related to the assessment of educational progress is available in Latin America, especially after the implementation of educational reforms during the last decades. This article aims to map how researchers use data of educational assessments conducted in Brazil, Chile and Argentina. The methodology used for identifying and selecting studies was a search for publications through the CAPES database, between 1994 and 2014. Using the keywords: "school performance", "rendimiento escolar" (in Spanish) and "rendimento escolar" (in Portuguese), SAEB, SIMCE and "National Operational Assessment"; 27 empirical studies using assessment data related to the three countries concerned were selected. The most frequently used technique in these articles was multilevel regression analysis. The review indicated that practically all of the selected articles analyzed the impact of factors associated to school performance. However, educational assessment data were also used to evaluate the role of schools in promoting equity and to estimate the impact of educational programs. The results of the present study allow the general conclusion that the revised articles supply information that can support policies that contribute the improvement of education.
\end{abstract}

Keywords: School performance, Educational assessment, Factors associated with school performance, School effect, Social program evaluation, Multilevel analysis, Elementary school, High school, Latin America.

*Contacto: lgvinha@gmail.com

issn: $1989-0397$

www.rinace.net/riee/

https://revistas.uam.es/riee
Recibido: 12 de mayo de 2016

$1^{\text {a }}$ Evaluación: 17 de junio de 2016

Aceptado: 1 de agosto de 2016 


\section{Introducción}

En las últimas décadas, la educación en América Latina ha recibido especial atención por parte de los gobiernos. Según Casassus (2001), las reformas en los sistemas educativos de los países latinoamericanos, iniciadas en la década de 1960, pueden ser divididas en dos ciclos. El primer ciclo tenía como objetivo la expansión de las redes educativas, apuntando hacia la ampliación del número de personas que ingresan en los sistemas. En el segundo, iniciado en la década de 1980, fueron propuestos cambios más complejos enfocados en la gestión de los sistemas y enfatizando la promoción de educación con calidad.

Actualmente, las consecuencias del primer ciclo de cambios se puede observar fácilmente en los indicadores educativos de los países y en evaluaciones internacionales. Por ejemplo, entre los países de América Latina y el Caribe, en promedio, 94\% de los niños en edad de estar en nivel primario están en la escuela, y se observa también mayor acceso a la educación secundaria en las últimas décadas (UNESCO, 2015). Se podría considerar que la universalización de la educación es una realidad más cercana y por lo tanto la preocupación principal pasa a ser la calidad y equidad de la educación en los países.

En el segundo ciclo, principalmente en la década de 1990, las reformas propuestas buscaban situar la educación como centro de la estrategia de los gobiernos. Fueron propuestos cambios de gestión basados en la descentralización y énfasis en la calidad y equidad, además de mejoras en los niveles de aprendizaje por medio de acciones coordinadas (Casassus, 2001). En especial, la descentralización de la educación impuso modificaciones en el control de las escuelas, antes centralizado en el Estado, y la incorporación de la iniciativa privada en la gestión. El Estado pasa a tener, predominantemente, la función de coordinación y reglamentación del sistema educativo (Krawczyk y Vieira, 2010).

En este contexto, las evaluaciones educativas a gran escala surgen como un instrumento fundamental para el nuevo papel asumido por el Estado. Estas evaluaciones son indispensables para la supervisión del sistema y el seguimiento de las acciones de mejora, asimismo, tienen un papel estratégico para la formulación de nuevas políticas y programas (Castro, 2000).

América Latina tiene por lo menos dos décadas de experiencia en evaluaciones educativas (Ferrer, 2006). Las principales evaluaciones realizadas en la región fueron creadas a finales de la década de 1980 e inicio de la década de 1990. Los estudiantes latinoamericanos participan también de evaluaciones internacionales. El LLECE ${ }^{1}$ evalúa la calidad de la educación en la enseñanza básica en América Latina y el Caribe. Algunos países de la región participan de las pruebas PISA (Programme for International Student Assessment), cuyo foco es el desempeño de estudiantes en la franja de los 15 años, edad en que se presupone el término de la educación básica obligatoria en la mayoría de los países.

\footnotetext{
${ }^{1}$ Laboratorio Latinoamericano de Evaluación de la Calidad de la Educación, Oficina Regional de la UNESCO (United Nations Educational, Scientific and Cultural Organization).
} 
Diversas evaluaciones importantes son realizadas a nivel nacional en la región: el EXCALE (Exámenes para la Calidad y el Logro Educativo) en México; el SABER (Sistema Nacional de Evaluación de la Calidad de la Educación) en Colombia; y el APRENDO (Sistema Nacional de Medición de Logros Académicos) en Ecuador. Además de iniciativas regionales como, por ejemplo, los sistemas de evaluación de los estados de Minas Gerais y São Paulo en Brasil y de la ciudad de Bogotá en Colombia (Ferrer, 2006). Por lo tanto, la disponibilidad de información acerca de educación es grande en la región.

En este contexto, el presente estudio tiene como objetivo evaluar cómo los investigadores han utilizado los datos de evaluaciones educativas desarrolladas en Brasil, Argentina y Chile. Se analiza cómo estos datos han sido utilizados y cuáles han sido los objetivos de las investigaciones, las técnicas de análisis utilizadas y los principales hallazgos reportados por los investigadores. Los tres países elegidos tienen en común el carácter descentralizado de las reformas educativas en la década de 1990 (Krawczyk y Vieira, 2006) y también la similitud de resultados en las evaluaciones de PISA (en particular para Brasil y Argentina). Los sistemas de evaluación elegidos de estos tres países tienen aún similitudes en alcance y ciclos evaluados, los detalles son presentados a continuación.

\subsection{Sistema Nacional de Evaluación de Educación Básica}

En Brasil, el SAEB (Sistema Nacional de Avaliação da Educação Básica) es el principal sistema de evaluación educativa. Las evaluaciones son realizadas cada dos años bajo la coordinación del INEP (Instituto Nacional de Estudos e Pesquisas Educacionais Anísio Teixeira). El SAEB tiene como principal objetivo evaluar la educación básica y contribuir para la mejora de su calidad y para la universalización del acceso a las escuelas, ofreciendo subsidios concretos para la formulación, reformulación y el control de las políticas públicas. Además, "busca también ofrecer datos e indicadores que posibiliten mayor comprensión de los factores que influyen en el desempeño de los alumnos en las áreas y años evaluados" (Cabral y Figueiredo, 2002, p. 1).

Las evaluaciones del SAEB se iniciaron en 1990 y sufrieron diversas modificaciones desde entonces. En las primeras ediciones fueron evaluados alumnos que cursaban $1^{\circ}, 3^{\circ}$, $5^{\circ}$ y $7^{\circ}$ año de educación primaria y, a partir de la edición de 1995, el foco pasó a ser los años $4^{\circ}$ y $8^{\circ}$ de educación primaria y $3^{\circ}$ de educación secundaria. En relación a las disciplinas evaluadas, las pruebas de Matemática y Lengua Portuguesa están presentes en todas las evaluaciones, sin embargo, otras disciplinas fueron evaluadas únicamente en algunas ediciones. Entre 1990 y 2001 fueron seleccionadas muestras de alumnos y a partir de 2005 se buscó evaluar a todos los alumnos matriculados en los años de interés.

Las pruebas por competencias utilizadas en el sistema se basan en el currículo mínimo de cada disciplina y asocian los contenidos curriculares a las competencias cognitivas utilizadas en el proceso de construcción del conocimiento (INEP, 2007). En las primeras ediciones las competencias de los alumnos eran calculadas en base a la teoría clásica de los tests, no obstante, a partir de la edición de 1995, los resultados pasaron a ser calculados a través de la Teoría de Respuesta al Ítem (TRI). La utilización de la TRI representó un gran avance en la evaluación, pues permitió la construcción de una escala única para los años evaluados y la clasificación de los alumnos en términos de desempeño (INEP, 2007). 
Las características de los alumnos y los factores contextuales son obtenidos a partir de la aplicación de diferentes cuestionarios. En el SAEB son aplicados cuatro cuestionarios distintos: para el alumno, el director, el profesor y la escuela. Estos instrumentos presentan una caracterización del nivel socioeconómico y cultural, aspectos familiares, formación de los profesionales, formas de gestión, prácticas pedagógicas e infraestructura de las escuelas.

\subsection{SIMCE - Sistema de Medición de Logros de Aprendizaje}

En Chile, el sistema de evaluación de desempeño escolar fue desarrollado con el objetivo de contribuir a mejorar la calidad y la equidad de la educación. Para ello, el desempeño de alumnos en diferentes áreas del currículo nacional es evaluado y relacionado con el contexto escolar y social en que ocurre el aprendizaje (Agencia de Calidad de la Educación, 2014). Las pruebas del SIMCE son aplicadas anualmente.

En el periodo entre 1990 y 1997 fueron evaluados en Matemática y Lenguaje y Comunicación todos los alumnos matriculados en el $4^{\circ}$ y $8^{\circ}$ año de educación primaria, y para las disciplinas de Ciencias, Historia y Geografía fueron aplicadas pruebas solo a una muestra de alumnos (Meckes, 2003). En este periodo los resultados eran expresados por medio del porcentaje de respuestas correctas, sin considerar la dificultad de las preguntas, lo que imposibilitaba la comparación entre los alumnos cursando años distintos y entre distintas versiones de la evaluación. En 1998, una serie de cambios fue implementada en el SIMCE, entre ellos la adecuación a un nuevo currículo y la utilización de la TRI para obtener las puntuaciones (Bravo, 2011). La utilización de la TRI posibilitó la comparación de resultados entre los años y también la diferenciación de niveles de desempeño de los estudiantes a partir de las puntuaciones obtenidas.

Hasta 2005 la aplicación de las pruebas del SIMCE se alternó entre los alumnos de $4^{\circ} \mathrm{y}$ $8^{\circ}$ grado de educación primaria y el $2^{\circ}$ grado de educación secundaria. A partir del 2006, los alumnos de $4^{\circ}$ grado pasaron a ser evaluados todos los años y la evaluación de los demás grados pasó a ser de forma alternada (Meckes, 2003). Cabe resaltar que se realizaron cambios importantes con el objetivo de dar seguimiento a cohortes de estudiantes. Con este cambio, en 2009, fue posible evaluar el progreso de un grupo de alumnos del $8^{\circ}$ grado que habían sido evaluados en el $4^{\circ}$ grado en 2005 . En el mismo periodo, se realizaron inversiones para aumentar el acceso a las pruebas, evaluar estudiantes con deficiencia visual y auditiva, incluir la evaluación de otras disciplinas y mejorar la divulgación de los resultados.

Además de las pruebas, se recopila información de profesores, estudiantes y padres o apoderados. En los cuestionarios respondidos por los estudiantes son evaluados aspectos generales de las escuelas, estrategias de aprendizaje dentro y fuera del aula, intereses, motivaciones y otros. En los cuestionarios de los padres están presentes asuntos relacionados al nivel educativo, renta y nivel de satisfacción con el establecimiento educativo. Los profesores responden a cuestiones relacionadas a su formación profesional y al contenido abordado durante el año.

\subsection{ONE - Operativo Nacional de Evaluación}

En Argentina, desde 1993, son realizadas las evaluaciones del sistema educativo argentino por medio del ONE. Este sistema es actualmente ejecutado por el DiNIECE (Dirección Nacional de Información y Evaluación de la Calidad Educativa), órgano 
ligado al Ministerio de Educación de la Nación Argentina y que tiene los siguientes propósitos: contribuir a la mejora de la calidad de la educación a través de la evaluación de desempeño de los estudiantes en diferentes niveles y áreas; y proveer información estadística, psicométrica y pedagógica a nivel nacional y provincial, a partir del análisis del aprendizaje de los alumnos y factores asociados, para subsidiar el desarrollo de políticas educacionales (DiNIECE, 2007).

De forma general, los estudiantes de $3^{\circ}$ y $6^{\circ}$ (finales de los ciclos de la educación primaria) y de $2^{\circ}$ y $3^{\circ}$ de educación secundaria son evaluados por el sistema. Los alumnos son evaluados por medio de pruebas de Lengua, Matemática, Ciencias Sociales y Ciencias Naturales con ítems alineados con el currículo nacional. La TRI también es utilizada para generar las puntuaciones de los alumnos, lo que posibilita la comparación entre años y periodos y el análisis de los ítems que componen las pruebas (DiNIECE, 2007).

Con relación a la periodicidad, es de notar que inicialmente se pretendía realizar evaluaciones anuales, pero dada la época de crisis en dicho país, esto no fue posible (Berardi, 2012). En el periodo de 1993 a 2000, las evaluaciones se dieron de forma ininterrumpida, retornando después de la crisis económica en el 2002 y 2003. A partir del 2005, las evaluaciones pasaron a ser realizadas bianualmente, con una nueva interrupción en el 2009.

Así como otros sistemas de evaluación, el ONE consiste en la aplicación de pruebas de conocimiento y cuestionarios contextuales. Los cuestionarios ofrecen informaciones relacionadas a los antecedentes académicos de los alumnos, nivel socioeconómico y educativo de la familia, motivación académica y percepciones sobre la institución, así como características de directores y docentes, prácticas pedagógicas, infraestructura y recursos de la escuela (DiNIECE, 2007).

Además del papel estratégico en la administración de los sistemas educativos de los países, las evaluaciones a gran escala son fuentes valiosas de información acerca del proceso de aprendizaje. Todavía, en muchas situaciones, los datos brutos oriundos de las evaluaciones no son suficientes para la comprensión del complejo proceso de aprendizaje, estos datos deben ser analizados y traducidos en informaciones útiles que puedan ser usadas para orientar acciones.

Los datos de las evaluaciones educativas han sido ampliamente utilizados en estudios de factores asociados con el rendimiento y eficacia escolar en diversos países. Esto se puede observar en algunas revisiones de la literatura. Por ejemplo, Teodorović (2009) presenta una extensa revisión de la literatura de trabajos acerca de la eficacia escolar en la cual apunta los principales factores asociados con el rendimiento académico teniendo en cuenta las características de las familias, la escuela y la estructura de las prácticas de enseñanza. Townsend (2007) presenta los principales resultados observados en la literatura con énfasis en los estudios correspondientes en los diferentes países y regiones.

Murillo (2007) también destaca el interés por los sistemas de evaluaciones educativas en todo el mundo y, en especial, en América Latina. El autor afirma que hubo un aumento significativo de investigación en los últimos años, consecuencia de la consolidación de la comunidad científica dedicada al tema en esta región. Los datos de evaluaciones 
educativas han sido usados por los investigadores latinoamericanos en los estudios de eficacia escolar, principalmente como herramienta para la identificación de factores asociados al desempeño, pero también para el análisis del efecto escuela y evaluaciones de programas.

\section{Método}

En este estudio se emplea el método presentado por Newman (2006). El proceso de revisión incluyó cuatro pasos: (1) la definición de los criterios de inclusión y exclusión; (2) la identificación sistemática de los estudios de acuerdo con los criterios establecidos; (3) la extracción de informaciones; y (4) la síntesis de los hallazgos de los estudios seleccionados.

\subsection{Definición de los criterios de inclusión y exclusión}

Los trabajos seleccionados deberían: ser estudios de naturaleza empírica sobre el uso de los datos de las evaluaciones; presentar resultados de evaluaciones a gran escala (nivel nacional o regional); utilizar como variable dependiente la competencia de los alumnos en Matemática, Lengua Portuguesa, Lengua Española, Ciencias o Literatura y Comprensión de Textos; analizar datos de desempeño escolar a nivel de alumnos; y utilizar técnicas inferenciales.

\subsection{Identificación de los estudios}

La localización y la selección de los estudios relevantes fueron realizadas utilizando la base de datos de revistas electrónicas de la CAPES (Coordenação de Aperfeiçoamento de Pessoal de Nivel Superior), disponible en la dirección electrónica http://www.periodicos.capes.gov.br. La consulta fue hecha en enero de 2015 considerando apenas los trabajos publicados en el periodo de 1994 y 2014. Fueron utilizadas las siguientes combinaciones de palabras clave: $S A E B$ y desempenho escolar; SAEB y school performance; SIMCE y rendimiento escolar; SIMCE y school performance; "Operativo Nacional de Evaluación” y Argentina. La inclusión de las expresiones "desempenho escolar", "school performance" y "rendimiento escolar" fue hecha tratando de reducir el número de referencias en las consultas relacionadas con SAEB y SIMCE. 376 referencias fueron obtenidas, siendo 208 relacionadas con SAEB, 158 con SIMCE y 10 con ONE. Después de la aplicación de los criterios de inclusión y exclusión, fueron seleccionados 27 estudios.

\subsection{Extracción de informaciones}

Se realizó una tabulación de estos trabajos, discriminando los siguientes ítems: autores, año, evaluación utilizada y edición, año escolar y disciplina evaluada, técnicas de análisis de datos, objetivos y resultados encontrados.

\section{Resultados}

La evaluación y el análisis de los contenidos son presentados en esta sección. Entre los 27 estudios seleccionados, 11 presentan el análisis de datos brasileños del SAEB, 11 se refieren a los datos del SIMCE de Chile y 5 utilizaron la evaluación ONE de Argentina. La Tabla 1 presenta algunas características generales de los estudios seleccionados. 
Tabla 1. Características generales de los trabajos seleccionados $(\mathrm{n}=27)$

\begin{tabular}{|c|c|c|c|c|c|c|}
\hline País & AutoRES/AÑo & BASE & $\begin{array}{c}\text { AÑo } \\
\text { ESCOLAR }\end{array}$ & $\begin{array}{c}\text { DISCIPLIN } \\
\text { A }\end{array}$ & ANÁLISIS & $\begin{array}{l}\text { OBJETIVO } \\
\text { PRINCIPAL }\end{array}$ \\
\hline $\mathrm{BR}$ & Soares y Alves (2003) & SAEB 2001 & $8^{\circ} \mathrm{EB}$ & MAT & $\mathrm{AM}$ & Equidad \\
\hline $\mathrm{BR}$ & Soares (2004) & SAEB 2001 & $8^{\circ} \mathrm{EB}$ & MAT & $\mathrm{AM}$ & Equidad \\
\hline $\mathrm{BR}$ & Andrade y Laros (2007) & SAEB 2001 & $3^{\circ} \mathrm{ES}$ & $\begin{array}{l}\text { MAT, } \\
\text { LP }\end{array}$ & $\mathrm{AM}$ & $\begin{array}{l}\text { Estudio de } \\
\text { factores }\end{array}$ \\
\hline $\mathrm{BR}$ & Franco et al. (2007) & SAEB 2001 & $4^{\circ} \mathrm{EB}$ & MAT & $\mathrm{AM}$ & Equidad \\
\hline $\mathrm{BR}$ & $\begin{array}{c}\text { Waltenberg y } \\
\text { Vandenberghe (2007) }\end{array}$ & SAEB 2001 & $8^{\circ} \mathrm{EB}$ & LP & $\mathrm{RC}$ & Equidad \\
\hline $\mathrm{BR}$ & $\begin{array}{c}\text { Gonçalves y França } \\
\text { (2008) }\end{array}$ & SAEB 2003 & $\begin{array}{l}4^{\circ} \mathrm{EB} \mathrm{y} \\
3^{\circ} \mathrm{ES}\end{array}$ & MAT & $\mathrm{AM}$ & Equidad \\
\hline $\mathrm{BR}$ & Wainer et al. (2008) & SAEB 2001 & $\begin{array}{c}4^{\circ}, 8^{\circ} \\
\mathrm{EB}, 3^{\circ} \\
\mathrm{ES}\end{array}$ & $\begin{array}{l}\text { MAT, } \\
\text { LP }\end{array}$ & IC & $\begin{array}{l}\text { Estudio de } \\
\text { factores }\end{array}$ \\
\hline $\mathrm{BR}$ & $\begin{array}{l}\text { Curi y Menezes-Filho } \\
\text { (2009) }\end{array}$ & SAEB 2003 & $\begin{array}{l}4^{\circ}, 8^{\circ} \\
\mathrm{EB}, 3^{\circ} \\
\mathrm{ES}\end{array}$ & MAT & $\mathrm{AR}$ & $\begin{array}{l}\text { Estudio de } \\
\text { factores }\end{array}$ \\
\hline $\mathrm{BR}$ & $\begin{array}{c}\text { Rodrigues, Rios-Neto y } \\
\text { Pinto (2011) }\end{array}$ & $\begin{array}{l}\text { SAEB } \\
1997 \mathrm{a} \\
2005\end{array}$ & $4^{\circ} \mathrm{EB}$ & MAT & $\mathrm{DC}$ & $\begin{array}{l}\text { Estudio de } \\
\text { factores }\end{array}$ \\
\hline $\mathrm{BR}$ & $\begin{array}{c}\text { Laros, Marciano y } \\
\text { Andrade } \\
(2012)\end{array}$ & SAEB 2001 & $3^{\text {a }} \mathrm{ES}$ & LP & $\mathrm{AM}$ & $\begin{array}{l}\text { Estudio de } \\
\text { factores }\end{array}$ \\
\hline $\mathrm{BR}$ & $\begin{array}{c}\text { Oliveira, Belluzzo y } \\
\text { Pazello (2013) }\end{array}$ & SAEB 2005 & $8^{\circ} \mathrm{EB}$ & MAT & $\mathrm{RC}$ & $\begin{array}{l}\text { Estudio de } \\
\text { factores }\end{array}$ \\
\hline $\mathrm{CH}$ & Mizala y Romaguera & $\begin{array}{l}\text { SIMCE } \\
1998\end{array}$ & $4^{\circ}, 8^{\circ} \mathrm{EB}$ & $\begin{array}{l}\text { MAT, } \\
\text { LE }\end{array}$ & $\mathrm{AM}, \mathrm{RC}$ & $\begin{array}{l}\text { Estudio de } \\
\text { factores }\end{array}$ \\
\hline $\mathrm{CH}$ & McEwan (2003) & $\begin{array}{l}\text { SIMCE } \\
1997\end{array}$ & $8^{\circ} \mathrm{EB}$ & $\begin{array}{l}\text { MAT, } \\
\text { LE }\end{array}$ & $\mathrm{AM}$ & $\begin{array}{l}\text { Estudio de } \\
\text { factores }\end{array}$ \\
\hline $\mathrm{CH}$ & Cádiz (2006) & $\begin{array}{l}\text { SIMCE } \\
1996\end{array}$ & $4^{\circ} \mathrm{EB}$ & MAT & AM & $\begin{array}{l}\text { Estudio de } \\
\text { factores }\end{array}$ \\
\hline $\mathrm{CH}$ & Beach et al. (2008) & $\begin{array}{c}\text { SIMCE } \\
20022005 \\
\text { y } 2006\end{array}$ & - & $\begin{array}{l}\text { MAT, } \\
\text { LE }\end{array}$ & $\mathrm{AM}$ & $\begin{array}{l}\text { Impacto de } \\
\text { programa }\end{array}$ \\
\hline $\mathrm{CH}$ & $\begin{array}{l}\text { Anand, Mizala y } \\
\text { Repetto (2009) }\end{array}$ & $\begin{array}{l}\text { SIMCE } \\
2002\end{array}$ & $4^{\circ} \mathrm{EB}$ & $\begin{array}{c}\text { MAT, } \\
\text { LE } \\
\text { Ciencias }\end{array}$ & $\mathrm{PP}$ & $\begin{array}{l}\text { Impacto de } \\
\text { programa }\end{array}$ \\
\hline $\mathrm{CH}$ & Bellei (2009) & $\begin{array}{l}\text { SIMCE } \\
2001 \mathrm{y} \\
2003\end{array}$ & $2^{\circ} \mathrm{ES}$ & $\begin{array}{l}\text { MAT, } \\
\text { LE }\end{array}$ & DD & $\begin{array}{l}\text { Impacto de } \\
\text { programa }\end{array}$ \\
\hline $\mathrm{CH}$ & $\begin{array}{l}\text { Contreras, Bustos y } \\
\text { Sepúlveda }(2010)\end{array}$ & $\begin{array}{l}\text { SIMCE } \\
2005\end{array}$ & $4^{\circ} \mathrm{EB}$ & $\begin{array}{l}\text { MAT, } \\
\text { LE }\end{array}$ & $\mathrm{AM}$ & $\begin{array}{l}\text { Estudio de } \\
\text { factores }\end{array}$ \\
\hline $\mathrm{CH}$ & $\begin{array}{l}\text { Carrasco y San Martín } \\
\qquad(2012)\end{array}$ & $\begin{array}{l}\text { SIMCE } \\
2004 \mathrm{y} \\
2006\end{array}$ & $8^{\circ} \mathrm{EB}$ & MAT & VA & $\begin{array}{l}\text { Impacto de } \\
\text { programa }\end{array}$ \\
\hline $\mathrm{CH}$ & Mizala y Torche (2012) & $\begin{array}{l}\text { SIMCE } \\
2002 \mathrm{y} \\
2004\end{array}$ & $4^{\circ}, 8^{\circ} \mathrm{EB}$ & $\begin{array}{l}\text { MAT, } \\
\text { LE }\end{array}$ & $\mathrm{AM}$ & $\begin{array}{l}\text { Estudio de } \\
\text { factores }\end{array}$ \\
\hline $\mathrm{CH}$ & $\begin{array}{c}\text { Thieme, Prior y } \\
\text { Tortosa-Ausina (2012) }\end{array}$ & $\begin{array}{l}\text { SIMCE } \\
2008\end{array}$ & $4^{\circ} \mathrm{EB}$ & $\begin{array}{l}\text { MAT, } \\
\text { LE }\end{array}$ & $\mathrm{DM}$ & $\begin{array}{l}\text { Estudio de } \\
\text { factores }\end{array}$ \\
\hline $\mathrm{CH}$ & Paredes (2014) & $\begin{array}{l}\text { SIMCE } \\
2009\end{array}$ & $8^{\circ} \mathrm{EB}$ & $\begin{array}{c}\text { MAT, } \\
\text { LE } \\
\text { Ciencias }\end{array}$ & $\mathrm{AM}$ & $\begin{array}{l}\text { Estudio de } \\
\text { factores }\end{array}$ \\
\hline
\end{tabular}


Tabla 1b. Características generales de los trabajos seleccionados (n=27) (continuación)

\begin{tabular}{|c|c|c|c|c|c|c|}
\hline AR & Cervini (2001) & ONE 1997 & $6^{\circ}, 7^{\circ} \mathrm{EB}$ & MAT & $\mathrm{AM}$ & $\begin{array}{l}\text { Estudio de } \\
\text { factores }\end{array}$ \\
\hline AR & Cervini (2005) & ONE 1997 & $7^{\circ} \mathrm{EB}$ & MAT & $\mathrm{AM}$ & $\begin{array}{l}\text { Estudio de } \\
\text { factores }\end{array}$ \\
\hline AR & Cervini (2010) & ONE 2000 & $\begin{array}{l}6^{\circ} \mathrm{EB}, \\
3^{\circ} \mathrm{ES}\end{array}$ & $\begin{array}{c}\text { MAT, } \\
\text { LE }\end{array}$ & $\mathrm{AM}$ & Efecto escuela \\
\hline AR & Cervini (2011) & ONE 2000 & $6^{\circ} \mathrm{EB}$ & MAT & $\mathrm{AM}$ & $\begin{array}{l}\text { Estudio de } \\
\text { factores }\end{array}$ \\
\hline AR & $\begin{array}{l}\text { Cervini, Dari y Quiroz } \\
(2013)\end{array}$ & ONE 2007 & $3^{\circ} \mathrm{ES}$ & MAT & $\mathrm{AM}$ & $\begin{array}{l}\text { Estudio de } \\
\text { factores }\end{array}$ \\
\hline
\end{tabular}

Notas: EB - Educación Básica; ES - Educación Secundaria; MAT - Matemática; LP - Lengua Portuguesa; LE - Lengua Española; AR - Análisis de Regresión; AM - Análisis de Regresión Multinivel; RC - Regresión Cuantílica; IC - Intervalos de Confianza; DC - Descomposición contrafactual; PP - Puntaje de Propensión; VA - Análisis de Valor Agregado.

Fuente: Elaboración propia.

Se puede observar en la Tabla 1 que los trabajos seleccionados fueron publicados entre 2001 y 2014, a pesar de que la búsqueda inicial consideró publicaciones entre 1994 y 2014. En 22 estudios fueron analizados datos de educación básica y en 8 estudios datos de educación secundaria (Beach et al. no especificaron el año analizado). La competencia en Matemática fue utilizada como variable criterio en 25 estudios y la competencia en Lengua portuguesa o española fue usada en 14 trabajos.

En la gran mayoría de estudios fueron utilizados enfoques que consideran la estructura jerárquica de los datos y, en general, los resultados fueron obtenidos a través de análisis de regresión multinivel (Tabla 1). La descomposición multinivel, el análisis de valor agregado y la técnica de diferencia en diferencias también fueron utilizadas dentro de la estructura jerárquica. Tres trabajos no utilizaron el enfoque multinivel, en estos estudios los resultados fueron obtenidos por medio de la aplicación de análisis de regresión (Curi y Meneses-Filho, 2009; Rodrigues, Rios-Neto y Pinto, 2011) y de construcción de intervalos de confianza (Wainer et al., 2008).

Los estudios fueron clasificados de acuerdo con el objetivo principal de la investigación (Tabla 1). Como parte de la clasificación, fueron identificados cuatro grupos de estudios. Un primer grupo formado por 17 estudios enfocados en identificar los factores asociados al desempeño escolar y/o cuantificar el efecto de los mismos. Dentro de este grupo, los estudios de Andrade y Laros (2007), Laros, Marciano y Andrade (2012), Thieme, Prior y Tortosa-Ausina (2012) y Mizala y Romaguera (2002) tenían objetivos más genéricos y apuntaban a estudiar los factores asociados al desempeño escolar con base en informaciones disponibles; los demás estudios focalizaron el efecto de uno o más factores específicos. El segundo grupo está formado por cinco trabajos que tienen por objetivo la evaluación del papel de las escuelas en la promoción de equidad educativa. El tercer grupo está formado por cuatro trabajos, cuyo foco es la evaluación del impacto de un programa del área educacional. Finalmente, la estimación del efecto escuela fue el objeto de estudio de Cervini (2010) y estaba entre los objetivos de otros trabajos.

Sin embargo, los grupos citados no son excluyentes, diversos estudios podrían haber sido clasificados en otros grupos dado los objetivos secundarios o resultados reportados. Por ejemplo, el trabajo de Franco et al. (2007) tiene como objetivo principal el análisis de promoción de equidad pero también identifica factores asociados al desempeño y estima 
el efecto escuela en las competencias de los alumnos. El análisis de los estudios mostró una gran intersección de resultados; por lo tanto, en esta revisión, se optó por la presentación de los resultados agrupándolos en secciones.

\subsection{Estudio de factores asociados}

El estudio de factores asociados al desempeño escolar está de alguna forma presente en todos los trabajos seleccionados. Algunos de estos trabajos se enfocan en la identificación y estudio de impacto de los factores; en otros, factores contextuales son manejados como variable de control en la comparación de grupos, estimación del efecto escuela o en la evaluación de impacto de programas. En esta sección, se indican los factores que presentan efecto significativo en el desempeño, independiente del objetivo del estudio.

La Tabla 2 presenta los factores a nivel de alumno más frecuentes en los estudios seleccionados. Los símbolos $+\mathrm{y}-$ indican el impacto positivo y negativo en el desempeño escolar, respectivamente.

Tabla 2. Variables en el nivel del alumno con efecto en el desempeño escolar

\begin{tabular}{|c|c|c|c|c|c|c|c|c|}
\hline País & $\operatorname{ESTUDIO}(\mathrm{N}=14)$ & NSE & $\begin{array}{l}\text { SEXO } \\
\text { MASCU } \\
\text { LINO }\end{array}$ & ETNIA & $\begin{array}{c}\text { DISTORSIÓN } \\
\text { EDAD/AÑO } \\
\text { ESCOLAR }\end{array}$ & $\begin{array}{l}\text { RECURSOS } \\
\text { CULTURALES }\end{array}$ & $\begin{array}{c}\text { EDUCACIÓN } \\
\text { DE LOS } \\
\text { PADRES }\end{array}$ & $\begin{array}{c}\text { NÚMERO } \\
\text { DE } \\
\text { LIBROS }\end{array}$ \\
\hline $\mathrm{BR}$ & $\begin{array}{c}\text { Soares y Alves } \\
(2003)\end{array}$ & & & - & & & & + \\
\hline $\mathrm{BR}$ & Soares (2004) & + & + & - & & & & + \\
\hline $\mathrm{BR}$ & $\begin{array}{l}\text { Andrade y Laros } \\
\qquad(2007)\end{array}$ & -* & + & - & - & + & & \\
\hline $\mathrm{BR}$ & $\begin{array}{c}\text { Franco et al. } \\
(2007)\end{array}$ & + & + & & - & & & \\
\hline $\mathrm{BR}$ & $\begin{array}{c}\text { Gonçalves y } \\
\text { França (2008) }\end{array}$ & + & + & - & - & & & \\
\hline $\mathrm{BR}$ & $\begin{array}{c}\text { Laros, Marciano } \\
\text { y Andrade } \\
(2012)\end{array}$ & & & - & - & + & + & \\
\hline $\mathrm{BR}$ & $\begin{array}{c}\text { Oliveira, } \\
\text { Belluzzo y } \\
\text { Pazello }(2013)\end{array}$ & + & + & - & - & & + & \\
\hline $\mathrm{CH}$ & Cádiz (2006) & -* & -+ & & & & + & + \\
\hline $\mathrm{CH}$ & $\begin{array}{l}\text { Beach et al. } \\
\text { (2008) }\end{array}$ & + & & & & & & \\
\hline $\mathrm{CH}$ & Bellei (2009) & + & -+ & & - & & & \\
\hline $\mathrm{CH}$ & $\begin{array}{l}\text { Mizala y Torche } \\
\text { (2012) }\end{array}$ & + & -+ & & - & & & + \\
\hline AR & Cervini (2001) & + & & & & + & & \\
\hline AR & Cervini (2005) & + & + & - & & + & & \\
\hline
\end{tabular}

El nivel socioeconómico (NSE), el sexo y la trayectoria de los alumnos son factores que se presentan con frecuencia en los estudios. De forma general, se puede afirmar que cuanto mayor el nivel socioeconómico del alumno, mejor es su desempeño, que los alumnos de sexo masculino tienen notas superiores en Matemática e inferiores en pruebas de Lengua (portuguesa o española) y que los alumnos con histórico de abandono 
o de repetición tienen desempeño inferior. La utilización de variables relacionadas a etnias es común en los artículos brasileños, estos estudios reportan que alumnos no blancos (con excepción de alumnos de origen asiático) tienen desempeño escolar promedio inferior. La Tabla 2 destaca, a su vez, el impacto positivo de factores relacionados al capital cultural de las familias (escolaridad de los padres, recursos culturales de las familias y número de libros en casa).

El efecto negativo del nivel socioeconómico de los alumnos observado en dos estudios de la Tabla 2 probablemente está relacionado con la utilización de esta variable agregada en el nivel de la escuela. Es importante destacar que en Andrade y Laros (2007) fue observado un impacto muy fuerte y positivo del nivel socioeconómico medio en las escuelas sobre el desempeño en Matemática. En McEwan (2003), el nivel socioeconómico medio también tiene impacto fuerte y positivo en el desempeño en Matemática y en el nivel del alumno, aunque significativo, el impacto observado es bajo.

Los estudiantes a los cuales les gusta la disciplina y hacen las tareas tienen mejores desempeños, según los estudios de la Tabla 3. Algunos autores verificaron también que las expectativas en relación a los estudios de los hijos están positivamente asociadas con el desempeño y que la participación de los padres está asociada al desempeño inferior, con excepción del resultado observado por Soares (2004). El efecto negativo de la participación de los padres, según Andrade y Laros (2007), puede deberse al hecho de que los padres de alumnos con problemas de aprendizaje acuden a las escuelas con mayor frecuencia y exigen hacer las tareas, así como tener resultados escolares positivos a sus hijos.

Tabla 3. Otras variables del nivel del alumno con efecto en el desempeño escolar

\begin{tabular}{|c|c|c|c|c|c|}
\hline País & $\operatorname{ESTUDIO}(\mathrm{N}=8)$ & $\begin{array}{c}\text { TAREA } \\
\text { ESCOLAR }\end{array}$ & $\begin{array}{l}\text { PARTICIPACIÓN } \\
\text { DE LOS PADRES }\end{array}$ & $\begin{array}{l}\text { GUSTO POR } \\
\text { LA DISCIPLINA }\end{array}$ & $\begin{array}{l}\text { EXPECTATIVA } \\
\text { DE LOS PADRES }\end{array}$ \\
\hline $\mathrm{BR}$ & Soares y Alves (2003) & + & & & \\
\hline $\mathrm{BR}$ & Soares (2004) & + & + & + & \\
\hline $\mathrm{BR}$ & Andrade y Laros (2007) & + & - & + & \\
\hline $\mathrm{BR}$ & Franco et al. (2007) & + & & & \\
\hline $\mathrm{BR}$ & $\begin{array}{c}\text { Laros, Marciano y Andrade } \\
\text { (2012) }\end{array}$ & + & - & + & \\
\hline $\mathrm{CH}$ & Beach et al. (2008) & & & & + \\
\hline $\mathrm{CH}$ & Bellei (2009) & & & & + \\
\hline
\end{tabular}

Fuente: Elaboración propia.

Más factores relacionados con los alumnos fueron evaluados por los investigadores. Por ejemplo, Curi y Menezes-Filho (2009) analizaron la relación entre la educación preescolar y el desempeño académico. En este estudio los autores apuntan que los alumnos que comenzaron a estudiar en el preescolar tuvieron, en promedio, mejor desempeño en la prueba de Matemática que los alumnos que comenzaron a estudiar a partir del primer año de la educación primaria. El impacto observado es mayor en los alumnos de $4^{\circ}$ año y permanece significativo en los de $8^{\circ}$ y $3^{\circ}$ de educación secundaria. La educación preescolar también es considerada en otros estudios. Mizala y Torche (2012) reportan el impacto positivo del preescolar en el desempeño de los alumnos del $4^{\circ}$ año de educación primaria y al final de la educación secundaria, respectivamente.

Wainer et al. (2008) tenían como objetivo principal el análisis de impacto del uso de computadoras en el desempeño escolar de alumnos de $4^{\circ}$ y $8^{\circ}$ año de educación primaria 
y $3^{\circ}$ de educación secundaria. Los autores verificaron un impacto negativo y significativo en las puntuaciones de los alumnos que usan computadoras frecuentemente, siendo que ese impacto es más acentuado entre los más jóvenes y con menos nivel socioeconómico. El uso de la computadora también presenta un efecto negativo en el desempeño de los alumnos en el estudio de Laros, Marciano y Andrade (2012).

El estudio de Cervini (2005) tiene como enfoque primero el análisis del efecto del trabajo fuera de casa en el desempeño de los alumnos. Este autor verificó el impacto negativo del trabajo fuera de casa en las notas obtenidas en Matemática, incluso después de la introducción de variables que miden el nivel socioeconómico y cultural de las familias. Verificó también que los alumnos que trabajaban cuatro horas o más, en lugares distantes de sus casas, tienen el peor desempeño en las pruebas de Matemática. Los efectos negativos de este factor también fueron verificados por Andrade y Laros (2007) y Laros, Marciano y Andrade (2012).

Otros trabajos realizados con los datos del sistema ONE de Argentina evalúan factores no explorados en estudios de otros países. Cervini (2001) y Cervini (2011) buscaron evaluar la relación entre la oportunidad de aprender y el rendimiento escolar medido por medio de pruebas de Matemática. A partir del cuestionario respondido por profesores, fueron identificadas variables relacionadas a la oportunidad de aprender, la cobertura del currículo vigente, la frecuencia de las tareas escolares y la importancia dada a la enseñanza de las competencias evaluadas en las pruebas. Con base en los resultados observados, el autor afirma que existe una fuerte relación entre la oportunidad de aprender y el rendimiento de los alumnos, y que esto está relacionado con la composición del alumnado y los recursos disponibles en la escuela, pero que la variable oportunidad de aprender permanece significativa después de la inclusión de esas variables. En Cervini Dari y Quiroz (2013), son analizados los efectos de diferentes aspectos del proceso escolar en el desempeño en Matemática, según la percepción de alumnos y directores. Los autores identificaron que existe relación entre estas variables con el desempeño, principalmente las relativas a la percepción de los alumnos. Incluso después de la introducción de variables de control, se verificó un efecto significativo de la motivación, esfuerzo y buena relación con el profesor sobre el desempeño de los estudiantes al final de la educación secundaria.

Numerosos factores relacionados a escuelas, profesores y directores fueron analizados en los estudios analizados. La Tabla 4 exhibe los principales factores que presentan impactos significativos.

En la Tabla 4 se observa que el tipo de escuela está presente en ocho estudios seleccionados. Estos trabajos relatan que las escuelas privadas presentan desempeño promedio superior en relación a las escuelas públicas. En los estudios chilenos, las escuelas privadas también presentan desempeño superior, sin embargo, las comparaciones fueron realizadas entre las escuelas subsidiadas en el sistema de voucher ${ }^{2}$

${ }^{2}$ En el sistema chileno, el gobierno subsidia la escuela elegida por los padres en proporción directa al número de estudiantes matriculados. El gobierno paga a cada escuela una unidad de subvención escolar voucher para cada niño que asiste a clases allí (Contreras, 2001). 
y las escuelas municipales, con excepción del trabajo de Mizala y Romaguera (2002), en el que la comparación también considera las escuelas privadas no subsidiadas.

Tabla 4. Principales variables en el nivel de la escuela con efecto en el desempeño

\begin{tabular}{|c|c|c|c|c|c|c|c|}
\hline País & $\operatorname{EstUdio~}(N=17)$ & $\begin{array}{l}\text { ESCUELA } \\
\text { PRIVADA }\end{array}$ & NSE & $\begin{array}{l}\text { ESTRUCTURA Y } \\
\text { EQUIPAMIENTO }\end{array}$ & $\begin{array}{l}\text { AMBIENTE } \\
\text { ESCOLAR }\end{array}$ & $\begin{array}{c}\text { EXPERIENCIA } \\
\text { DEL } \\
\text { PROFESOR }\end{array}$ & $\begin{array}{c}\text { SALARIO } \\
\text { DEL } \\
\text { PROFESOR }\end{array}$ \\
\hline $\mathrm{BR}$ & Soares y Alves (2003) & & & + & & & + \\
\hline $\mathrm{BR}$ & Soares $(2004)$ & + & + & + & & & + \\
\hline $\mathrm{BR}$ & $\begin{array}{c}\text { Andrade y Laros } \\
(2007)\end{array}$ & & + & + & + & + & \\
\hline $\mathrm{BR}$ & Franco et al. (2007) & & + & + & + & & \\
\hline $\mathrm{BR}$ & $\begin{array}{c}\text { Waltenberg y } \\
\text { Vandenberghe (2007) }\end{array}$ & & & & & + & \\
\hline $\mathrm{BR}$ & $\begin{array}{c}\text { Gonçalves y França } \\
\text { (2008) }\end{array}$ & + & & + & & & + \\
\hline $\mathrm{BR}$ & $\begin{array}{l}\text { Oliveira, Belluzzo y } \\
\text { Pazello (2013) }\end{array}$ & + & & + & & & \\
\hline $\mathrm{CH}$ & $\begin{array}{c}\text { Mizala y Romaguera } \\
(2002)\end{array}$ & + & + & & & + & \\
\hline $\mathrm{CH}$ & McEwan (2003) & & + & & & & \\
\hline $\mathrm{CH}$ & Cádiz (2006) & + & + & & & & \\
\hline $\mathrm{CH}$ & Beach et al. (2008) & & + & & & & \\
\hline $\mathrm{CH}$ & Bellei (2009) & + & + & & & & \\
\hline $\mathrm{CH}$ & $\begin{array}{l}\text { Mizala y Torche } \\
\text { (2012) }\end{array}$ & + & & & & + & \\
\hline $\mathrm{AR}$ & Cervini (2001) & & + & + & & & \\
\hline AR & Cervini (2011) & & & & + & & \\
\hline $\mathrm{AR}$ & $\begin{array}{c}\text { Cervini, Dari y Quiroz } \\
(2013)\end{array}$ & & + & + & & & \\
\hline
\end{tabular}

Fuente: Elaboración propia.

El estudio de Oliveira, Belluzzo y Pazello (2013) profundiza el análisis de la diferencia entre las escuelas privadas y públicas en Brasil. Además de identificar un desempeño promedio significativamente mayor entre los alumnos de las escuelas privadas, los autores resaltan la importancia de las condiciones de las familias en comparación al efecto de otras variables, incluyendo características de las escuelas. La descomposición realizada en el estudio apunta que los alumnos con bajo rendimiento de las escuelas públicas tendrían resultados aún peores si estudiasen en escuelas privadas.

El uso del nivel socioeconómico promedio de la escuela es frecuente en los estudios analizados (Tabla 4), en general este factor es utilizado como variable de control. En los estudios de desempeño académico basados en datos transversales, se hace necesaria la utilización de variables que retiren la influencia de composición de las escuelas, frecuentemente el nivel socioeconómico agregado es utilizado para este fin. Los estudios presentados en la Tabla 4 también mencionan el impacto positivo de la infraestructura de las escuelas, el ambiente escolar favorable, la experiencia y el salario de los profesores.

Además del nivel socioeconómico promedio de los alumnos, otras variables agregadas fueron utilizadas en el nivel de la escuela, entre ellas: alumnos que trabajan (Andrade y Laros, 2007; Cervini, Dari y Quiroz, 2013; Laros, Marciano y Andrade, 2012); alumnos repitentes (Andrade y Laros, 2007; Cervini, Dari y Quiroz, 2013); nivel de escolaridad de los padres (Cervini, 2005; McEwan, 2003; Oliveira, Belluzzo y Pazello, 2013); recursos culturales (Andrade y Laros, 2007; Cervini, 2005); tareas escolares (Andrade y Laros, 
2007); gusto por la disciplina (Andrade y Laros, 2007); sexo (Oliveira, Belluzzo y Pazello, 2013); cantidad de libros (Cervini, Dari y Quiroz, 2013); y número de personas en casa (Cervini, 2005).

Los investigadores relataron la importancia de otros factores relacionados a los profesores: etnia (Oliveira, Belluzzo y Pazello, 2013); formación (Oliveira, Belluzzo y Pazello, 2013; Soares, 2004; Soares y Alves, 2003); demanda de tareas escolares (Franco et al., 2007); resolución de problemas en aula (Franco et al., 2007); relación profesor/alumno (Mizala y Romaguera, 2002); expectativa en relación a los alumnos, contenido impartido, relación con el director y funcionarios, percepción de seguridad y problemas en las escuelas (Soares, 2004). Entre las características de los directores que tuvieron impacto en el desempeño, fueron destacadas: participación (Soares y Alves, 2003); perfil de liderazgo (Franco et al., 2007); percepción en relación al compromiso de los profesores y problemas internos y externos (Soares, 2004); y relación con la comunidad (Gonçalves y França, 2008). Así mismo, en relación a las escuelas, se verificó el efecto de tamaño (Bellei, 2009; Cádiz, 2006) y de la presencia de biblioteca en las aulas (Franco et al., 2007).

El impacto de la interacción entre variables en el contexto escolar también fue analizado por los investigadores. Por ejemplo, el estudio de Paredes (2014) tuvo como objetivo evaluar el efecto del género del profesor en el desempeño de los alumnos. En este trabajo fueron utilizadas variables de interacción indicando el emparejamiento de género entre los alumnos en los modelos. La autora verificó que existe un efecto significativo entre el género del profesor y el rendimiento de las alumnas, en promedio, los resultados son mejores cuando la disciplina es impartida por una profesora.

Laros, Marciano y Andrade (2012) identificaron efectos de interacción entre las variables atraso escolar y el porcentaje de repetición de la clase y entre atraso y el porcentaje de alumnos que trabajan en la clase. Según los autores, se puede afirmar que el efecto de atraso escolar es mayor cuando el alumno está en una clase con mayor porcentaje de repitentes o de alumnos que trabajan.

El efecto de interacción entre el tipo de escuela y el nivel socioeconómico fue evaluado por Contreras, Bustos y Sepúlveda (2010). Los resultados observados indicaron que "las escuelas públicas con menor nivel socioeconómico presentaron rendimiento académico superior a las escuelas privadas con el mismo tipo de alumnos" (p. 1357). Mizala y Romaguera (2002) también evaluaron el efecto de interacción entre el nivel socioeconómico y el tipo de escuela y enfatizan que la asociación entre el nivel socioeconómico de los alumnos y el desempeño escolar es más débil para las escuelas privadas sin subsidio del gobierno, en comparación con las escuelas públicas y las escuelas privadas subsidiadas.

Finalmente, Rodrigues, Rios-Neto y Pinto (2011) también presentan un estudio de factores asociados; no obstante, difiere de los demás en cuanto al periodo analizado. Los autores evaluaron el impacto del nivel socioeconómico en el desempeño de los alumnos considerando las ediciones de 1997 a 2005 del SAEB. Los principales resultados observados indican que el aumento en el número de matrículas en el final de los años 1990 en Brasil "redujo el nivel socioeconómico promedio de los alumnos y colaboró para disminuir el promedio y elevar la desigualdad entre los resultados escolares” y al mismo 
tiempo "hubo reducción en la sensibilidad del desempeño escolar en el nivel socioeconómico” (Rodrigues, Rios-Neto y Pinto, 2011, p. 26).

\title{
3.2. Estudios de equidad
}

Una serie de artículos de investigadores brasileños presenta análisis de la equidad del sistema educativo. Esos estudios tienen como objetivo evaluar en qué grado las escuelas brasileñas son capaces de reducir las desigualdades de desempeño académico cuando son comparados grupos de acuerdo con el nivel socioeconómico, la etnia y el sexo.

El estudio de Franco et al. (2007) tuvo por objetivo identificar las características escolares asociadas tanto al aumento del desempeño promedio de las escuelas cuanto a moderar el efecto del origen socioeconómico de los alumnos sobre sus resultados escolares. A partir de los datos de los alumnos de $4^{\circ}$ año de educación primaria, los autores verificaron que factores escolares como la demanda de tareas escolares y el clima disciplinar tienen efectos positivos en el desempeño promedio de las escuelas, pero también afectan la relación entre el desempeño y el nivel socioeconómico, aumentando la desigualdad intra-escolar.

Gonçalves y França (2008) buscaron verificar cómo la desigualdad social es reproducida en el sistema educativo y cómo identificar factores que podrían actuar como instrumento de ruptura entre el bajo nivel socioeconómico de la familia y el bajo desempeño escolar de los hijos. El estudio se basó en los datos del SAEB 2003, considerando los alumnos que frecuentaban el $4^{\circ}$ año de educación primaria y el $3^{\circ}$ año de educación secundaria, en modelos con tres niveles de agregación (alumnos, escuelas y estados). Los autores identificaron los factores asociados al desempeño académico promedio de los alumnos (ver Tablas 2 y 4), pero poco pudieron afirmar en términos de la reducción de las desigualdades, principalmente para los alumnos del $4^{\circ}$ año de la educación primaria. Para los datos relativos al $3^{\circ}$ año de la educación secundaria, los autores identificaron el efecto de la infraestructura de la escuela y la actuación de concejos escolares en la reducción del impacto del nivel socioeconómico en su desempeño. Por tanto, los autores concluyen que:

\begin{abstract}
En la educación secundaria, el incentivo para la constitución de concejos escolares e inversión en la infraestructura de la escuela son políticas que se pueden mostrar eficaces en el sentido de elevar el nivel de equidad del sistema educativo y, por lo tanto, promover una igualdad de oportunidades, independientemente del trasfondo cultural y económico del estudiante. (p. 45)
\end{abstract}

El análisis del impacto de las políticas y prácticas escolares en la promoción de equidad es realizado por Soares y Alves (2003). Los autores analizaron varios indicadores asociados a la etnia de los alumnos y verificaron que existen diferencias significativas de desempeño entre blancos y pardos y entre blancos y negros. Los resultados observados indican que los factores escolares evaluados tienden a aumentar la desigualdad. Por ejemplo, según los autores "las condiciones escolares positivas se potencializan cuando se refiere a los alumnos blancos, produciendo una espiral favorable que los impulsa mucho más que a los alumnos negros y pardos” (p. 36). Sin embargo, algunos resultados indican que la equidad entre blancos y pardos es una realidad más cercana que entre blancos y negros.

El trabajo de Soares (2004), además de presentar una síntesis de los resultados de los alumnos del SAEB 2001 en general, amplía la discusión sobre equidad considerando la 
desigualdad asociada al género, la etnia y el nivel socioeconómico. El autor identificó que características de profesores y condiciones de las escuelas están relacionadas con la desigualdad entre los grupos. Además, los factores familiares están asociados al aumento de las diferencias entre los grupos formados por la etnia y el nivel socioeconómico.

El estudio presentado por Waltenberg y Vandenberghe (2007) está asociado con la reducción de la desigualdad de desempeño entre grupos; sin embargo, se diferencia de los demás una vez que presenta una simulación de asignación de recursos. Según los autores, el artículo presenta una aplicación de la teoría de igualdad de oportunidades en educación de Roemer (1998), utilizando los datos brasileños del SAEB. El objetivo es proponer una reasignación de inversión en educación necesaria para igualar las puntuaciones de los alumnos de diferentes niveles socioeconómicos. Como resultado los autores sugieren modificaciones en la asignación de recursos que podrían disminuir las diferencias de desempeño, a pesar de reconocer que este tipo de propuesta puede ser difícil de ser implementada.

\subsection{Análisis de impacto de programas}

Cuatro estudios seleccionados tienen en común el uso de los datos del SIMCE para evaluar el impacto de programas implementados en la red educativa de Chile.

Bellei (2009) evaluó el impacto de la escuela en período integral a partir de los datos del SIMCE del 2001 y el 2003. Fue utilizada la técnica de diferencia en diferencias a partir de las informaciones acerca de los alumnos y las escuelas. Los resultados fueron obtenidos por la comparación del desempeño del grupo de tratamiento (formado por los alumnos que frecuentaban escuelas en período integral) y del grupo de control (compuesto por los alumnos que frecuentaban escuelas solamente en un período). El autor encontró que las escuelas en tiempo integral tienen un impacto positivo y significativo en el desempeño de los alumnos de educación primaria en Matemática y Lengua Española, antes y después de la entrada de las variables de control. Los resultados apuntaron también que el impacto del programa es mayor entre alumnos de la zona rural y alumnos con mejores desempeños.

En Anand, Mizala y Repetto (2009) es realizada la evaluación del efecto del sistema de voucher en el desempeño académico de los estudiantes de baja renta. La técnica utilizada en este caso es el emparejamiento por puntaje de propensión, aplicada a los datos de los alumnos del $4^{\circ}$ año de la edición del 2002 del SIMCE. La información de los cuestionarios de indicadores contextuales respondidos por alumnos y padres fue utilizada para el cálculo de los puntajes de propensión. Se verificó que los alumnos de baja renta de las escuelas subsidiadas tienen desempeño promedio superior a aquellos que frecuentan las escuelas públicas, sin embargo, no fue detectada una diferencia significativa entre escuelas particulares y escuelas subsidiadas para ese perfil de alumnos.

El impacto de un programa de apoyo a escuelas consideradas críticas (Programa de Escuelas Críticas) es evaluado en Beach et al. (2008). El análisis fue realizado con la comparación del desempeño de los alumnos de las escuelas que tuvieron intervención, con alumnos de escuelas de un grupo de control. Este grupo de control fue obtenido por emparejamiento a partir de características de las escuelas. Estos datos fueron analizados a través de modelos multinivel una vez que, según los autores, a pesar de que la muestra está compuesta por escuelas pobres, persistía la influencia del nivel sociocultural de las 
familias en los resultados; por tanto, para estimar el impacto del programa fue necesario el control de la influencia de variables externas a la escuela. Se observó un progreso más acentuado en las escuelas que tuvieron intervención, principalmente en Matemática y en Ciencias, y que el efecto del programa es mayor entre las escuelas con mayor vulnerabilidad social.

El sistema de voucher es evaluado por Carrasco y San Martin (2012) por medio de un análisis de valor agregado utilizando datos longitudinales. Fueron utilizadas informaciones de los alumnos en las ediciones de 2004 y 2006 del SIMCE. Los mismos alumnos son evaluados en dos momentos distintos y el impacto del sistema es medido en cuanto las escuelas agregan valor en términos de desempeño. El modelo utilizado también considera la estructura jerárquica de los datos una vez que son utilizadas características de alumnos, padres y escuelas. Los autores no verificaron diferencias entre las escuelas y afirmaron que el desempeño de la escuela, medido en términos de valor agregado, es independiente de la naturaleza administrativa (escuelas públicas y escuelas subsidiadas).

\subsection{Estimación del efecto escuela}

El uso de los datos de las evaluaciones para la estimación del efecto escuela fue el enfoque principal en el estudio de Cervini (2010) y está presente en otros estudios analizados (Tabla 5). Cervini (2010) buscó estimar el efecto escuela bruto con diferentes niveles de agregación y el efecto escuela obtenido después del ingreso de variables de control de alumnos y de composición de las clases. Cuando son considerados únicamente, apenas dos niveles de agregación, escuela y alumno, el efecto escuela observado para Matemática es de $32,3 \%$ en la educación primaria y 43,3\% en la secundaria, y para el desempeño en Lengua Española el efecto escuela es 30,7\% en primaria y 37,8 en la educación secundaria (Tabla 5). Cuando es considerada la descomposición en más niveles de agregación (alumno, clase, escuela y provincia) el efecto escuela cae y queda en torno al $23 \%$ para las dos disciplinas tanto en la educación primaria como en la secundaria. El autor también estima el efecto escuela después de la entrada de variables de control. Son utilizados el nivel socioeconómico de los alumnos, la repetición y el sexo como variables de composición de la escuela y el efecto escuela neto encontrado queda en torno al 8,6\% para las disciplinas y etapas de educación analizadas.

La Tabla 5 también presenta el efecto escuela estimado en otros trabajos. Una vez que se analizan datos de diferentes etapas de educación, dos disciplinas distintas y en países diferentes, es esperado que el efecto escuela sufra una fuerte variación. De forma general, se puede observar que el efecto escuela estimado para la educación básica en Chile presenta los menores valores en torno al $22 \%$. Algunos estudios presentan el efecto escuela líquido después de la inclusión de variables de composición del alumnado. En general, la inclusión de esas variables reduce considerablemente el valor estimado para el efecto escuela; entretanto, vale resaltar que las variables utilizadas como control varían entre los estudios. Andrade y Laros (2007) utilizan el nivel socioeconómico, el sexo y la etnia, tanto al nivel del alumno cuanto agregando las escuelas; Laros, Marciano

\footnotetext{
${ }^{3}$ Efecto escuela es el porcentaje de variación en el rendimiento de los alumnos debido a las características procesuales del centro en el que están escolarizados (Murillo, 2005).
} 
y Andrade (2012) usan el nivel socioeconómico, el sexo y la escolaridad de la madre también en los dos niveles; y Cádiz (2006) utiliza el tipo de escuela y el nivel socioeconómico promedio de las escuelas.

Tabla 5. El efecto escuela relatado en los trabajos

\begin{tabular}{|c|c|c|c|c|c|c|}
\hline PaÍs & $\operatorname{EsTUDIO}(\mathrm{N}=12)$ & BASE & $\begin{array}{c}\text { AÑo } \\
\text { ESCOLAR }\end{array}$ & DISCIPLINA & $\begin{array}{c}\text { EFECTO } \\
\text { ESCUELA } \\
\text { BRUTO } \\
\end{array}$ & $\begin{array}{c}\text { EFECTO } \\
\text { ESCUELA } \\
\text { NETO } \\
\end{array}$ \\
\hline $\mathrm{BR}$ & Andrade y Laros (2007) & SAEB 2001 & $3^{\circ} \mathrm{ES}$ & MAT, LP & $46,0 \%$ & $17,0 \%$ \\
\hline $\mathrm{BR}$ & Franco et al. (2007) & SAEB 2001 & $4^{\circ} \mathrm{EB}$ & MAT & $39,0 \%$ & \\
\hline BR & Gonçalves y França (2008) & SAEB 2003 & $\begin{array}{l}4^{\circ} \mathrm{EB} \\
3^{\circ} \mathrm{ES}\end{array}$ & MAT & $\begin{array}{l}30,7 \% * * \\
44,5 \% * *\end{array}$ & \\
\hline $\mathrm{BR}$ & $\begin{array}{l}\text { Laros, Marciano y Andrade } \\
\qquad(2012)\end{array}$ & SAEB 2001 & $3^{\circ} \mathrm{ES}$ & LP & $33,0 \%$ & $12,0 \%$ \\
\hline $\mathrm{CH}$ & Cádiz (2006) & $\begin{array}{l}\text { SIMCE } \\
1996\end{array}$ & $4^{\circ} \mathrm{EB}$ & MAT & $24,0 \%$ & $19,0 \%$ \\
\hline $\mathrm{CH}$ & Mizala y Torche (2012) & $\begin{array}{l}\text { SIMCE } \\
2002 / 04\end{array}$ & $\begin{array}{l}4^{\circ} \mathrm{EB} \\
8 \mathrm{a} \mathrm{EB}\end{array}$ & $\begin{array}{l}\text { MAT } \\
\text { LE } \\
\text { MAT } \\
\text { LE }\end{array}$ & $\begin{array}{l}21,4 \% \\
21,6 \% \\
22,8 \% \\
19,4 \%\end{array}$ & \\
\hline AR & Cervini (2001) & ONE 1997 & $6^{\circ}, 7^{\circ} \mathrm{EB}$ & MAT & $44,0 \%$ & \\
\hline AR & Cervini (2005) & ONE 1997 & $7^{\circ} \mathrm{EB}$ & MAT & $45,9 \%$ & \\
\hline AR & Cervini (2010) & ONE 2000 & $4^{\circ} \mathrm{EB}$ & MAT & $32,3 \%$ & $9,4 \%$ \\
\hline AR & Cervini (2011) & $\begin{array}{l}\mathrm{CFSNF}^{*} 98 \\
\text { ONE } 2000\end{array}$ & $\begin{array}{l}3^{\circ} \mathrm{ES} \\
6^{\circ} \mathrm{EB}\end{array}$ & $\begin{array}{l}\text { LE } \\
\text { MAT } \\
\text { LE } \\
\text { MAT }\end{array}$ & $\begin{array}{c}30,7 \% \\
43,3 \% \\
37,8 \% \\
36,2 \% * *\end{array}$ & $\begin{array}{l}8,4 \% \\
8,7 \% \\
8,1 \%\end{array}$ \\
\hline AR & $\begin{array}{c}\text { Cervini, Dari y Quiroz } \\
(2013)\end{array}$ & ONE 2007 & $3^{\circ} \mathrm{ES}$ & MAT & $34,1 \% * *$ & \\
\hline
\end{tabular}

Notas: * Censo Nacional de Finalización del Nivel Secundario.

** La descomposición de la variación incluye la provincia o estado.

Fuente: Elaboración propia.

\section{Conclusiones y discusión}

Este artículo presenta una revisión de literatura sobre el uso de los datos de las evaluaciones educativas a gran escala de Brasil, Chile y Argentina. En esta revisión se encontró que los resultados se pueden clasificar en cuatro grupos: los estudios de factores asociados con el rendimiento, los estudios de equidad, los programas de análisis de impacto y la estimación del efecto de la escuela. Las técnicas utilizadas generalmente consideran la estructura jerárquica de las poblaciones estudiadas, especialmente el análisis de regresión multinivel.

En general, los objetivos y relatos de los estudios seleccionados están alineados con el análisis de Murillo (2007) en la investigación sobre la eficacia escolar en América Latina. El autor enfatiza que en una gran parte de los estudios realizados los investigadores están interesados en analizar el impacto de los factores asociados al rendimiento escolar. En este trabajo, el autor también analiza los estudios realizados en los siguientes grupos o líneas de investigación: estudios ad hoc de la eficacia escolar; uso de evaluaciones de datos secundarios de sistemas educativos; estudios de efecto escuela, valoración de programas de mejora en las escuelas; estudios dirigidos a la comprensión de la relación entre los factores escolares y el rendimiento de los estudiantes; y el análisis de la cultura escolar bajo un enfoque etnográfico. Nótese que los objetivos y los resultados 
observados en el presente estudio coinciden con algunas líneas de investigación designados por Murillo (2007).

El impacto de algunos factores relacionados con los estudiantes es común entre los estudios revisados, con énfasis en el nivel socioeconómico. Sin embargo, vale la pena mencionar algunas diferencias en la forma de medir este factor. Las medidas propuestas por los autores varían considerablemente, tanto en variables como en las técnicas utilizadas en la construcción del indicador. Algunos ejemplos: Cervini (2001) utiliza un indicador compuesto por los bienes de uso durable de los hogares y la escolarización de los padres; Franco et al. (2007) utilizan el TRI de los ítems del cuestionario de los estudiantes del SAEB; Soares y Alves (2003) hacen uso de una metodología propuesta en PISA mediante análisis factorial; en Bellei (2009) y Anand, Mizala y Repeto (2009) fue utilizada una variable de ingresos familiares recogidos en los cuestionarios de los padres.

La estimación del efecto escuela también fue parte de los estudios, pero algunos resultados reportados deben interpretarse con cautela. De acuerdo con Soares y Candian (2007) el efecto escuela solo podría ser estimado a partir de datos longitudinales en la comparación entre el nivel de desempeño del estudiante en el momento de entrada y de salida de la escuela. Los autores también señalan que la estimación de este efecto a partir de datos transversales se podría ver afectada por la diferencia de composición de las escuelas y, por lo tanto, el efecto estimado no solo refleja la acción de la escuela. Sin embargo, estudios longitudinales a gran escala son raros. Una alternativa utilizada por los investigadores es la estimación del efecto escuela a partir de datos transversales con la introducción de variables de control en los modelos. Con la introducción de estas variables es posible observar otras fuentes de variabilidad entre las escuelas, además de las características de composición del alumnado (Andrade y Laros, 2007).

Con base en los resultados de este estudio, se puede observar que los datos de las evaluaciones han sido usados por los investigadores para el estudio de la relación entre el desempeño y el contexto escolar, que corresponde a uno de los objetivos principales de los sistemas de evaluación de la educación. Gracias a estos estudios se puede producir información a ser utilizada para mejorar la enseñanza y apoyar intervenciones. Del mismo modo, hubo un aumento en el número de artículos científicos en el área, como es observado por Murillo (2007), con mayor frecuencia de publicaciones en los últimos años del período analizado (20 de los 27 estudios fueron publicados a partir del año 2007).

El presente estudio busca contribuir a la comprensión del complejo proceso de aprendizaje a través del análisis de datos de evaluaciones educativas a gran escala. Se entiende que estos datos deben ser utilizados en la elaboración de políticas y programas educativos, para que las acciones sean eficaces en la mejora de la educación y la reducción de la desigualdad educativa. Se cree también que los hallazgos presentados pueden ser útiles para otros investigadores de la región y guiar nuevas investigaciones, una vez que los sistemas educativos son similares en varios aspectos. Algunos de los resultados destacados en este artículo ya se han reportado en otros estudios, con todo el trabajo presenta una comparación detallada de hallazgos de investigaciones realizadas en tres países.

Por último, este estudio tiene limitaciones en relación a su alcance. Se llevó a cabo la búsqueda de artículos en tan solo una base de datos de revistas científicas y esto representa una limitación, a pesar de que la CAPES cuente con gran cantidad de 
publicaciones. Fueron utilizadas como palabras clave el rendimiento escolar y el nombre de las evaluaciones y las evaluaciones utilizadas son relacionadas a apenas tres países de América Latina. Se sugiere que futuras investigaciones busquen: utilizar nuevas fuentes de información, otras bases de datos y diferentes tipos de publicaciones como libros, informes técnicos, tesis de maestrías y doctorados; utilizar, en las búsquedas, más palabras clave como "efecto escuela", "eficacia escolar", "evaluación de programas sociales” y "equidad”; y, además, analizar la producción de otros países de la región que tengan también importantes sistemas de evaluación escolar.

\section{Referencias}

Agencia de Calidad de la Educación. (2014). Informe Técnico SIMCE 2013. Recuperado de http://archivos.agenciaeducacion.cl/documentos-web/Informe_TecnicoSimce_2013.pdf

Anand, P., Mizala, A. y Repetto, A. (2009). Using school scholarships to estimate the effect of private education on the academic achievement of low income students in Chile. Economics of Education Review, 28, 370-381. doi:10.1016/j.econedurev.2008.03.005

Andrade, J. M. y Laros, J. A. (2007). Fatores associados ao desempenho escolar: Um estudo multinível com dados do SAEB/2001. Psicologia: Teoria e Pesquisa, 23(1), 33-42. doi:10.1590/s0102-37722007000100005

Beach, P. V., Eltit, P. C., Gonçalves, F. D. y Araya, M. M. (2008). Análisis del cambio en los niveles de logros de escuelas de alta vulnerabilidad social. Estudios Pedagógicos, 34(2), 179191. doi:10.4067/s0718-07052008000200011

Bellei, C. (2009). Does lengthening the school day increase students' academic achievement? Results from a natural experiment in Chile. Economics of Education Review, 28(5), 629-640. doi:10.1016/j.econedurev.2009.01.008

Berardi, R. (2012). Los problemas de implementación del Operativo Nacional de Evaluación 2010 (Tesis de Licenciatura, Escuela de Educación, Universidad de San Andrés, Vitoria).

Bravo, J. (2011). SIMCE: Pasado, presente y futuro del Sistema Nacional de Educación. Estudios Públicos, 123, 189-210.

Cabral, A. B. e Figueiredo, M. Z. (Coords.). (2002). SAEB - 2001: Novas perspectivas. Brasília: Instituto Nacional de Estudos e Pesquisas Educacionais.

Cádiz, J. (2006). Reanalyzing fourth grade math student achievement in Chile: Applying hierarchical linear models (HLMS). RELIEVE, 12(1), 75-91.

Carrasco, A. y San Martin, E. (2012). Voucher system and school effectiveness: Reassessing school performance difference and parental choice decision making. Estudios de Economía, 39(2), 123-141. doi:10.4067/s0718-52862012000200002

Casassus, J. (2001). A reforma educacional na América Latina no contexto da globalização. Cadernos de Pesquisa, (114), 7-28. doi:10.1590/s0100-15742001000300001

Castro, M. H. G. (2000). Sistemas nacionais de avaliação e de informações educacionais. São Paulo Perspectiva, 14(1), 121-128. doi:10.1590/s0102-88392000000100014

Cervini, R. (2001). Efecto de la "oportunidad de aprender" sobre el logro en matemáticas en la educación básica argentina. Revista Electrónica de Investigación Educativa, 3(2), 1-22.

Cervini, R. (2005). Trabajo infantil urbano y logro en matemáticas de la educación básica. Un modelo de dos niveles. Revista Mexicana de Investigación Educativa, 10(25), 451-480. 
Cervini, R. (2010). El 'Efecto escuela' en la educación primaria y secundaria: El caso de Argentina. Revista Iberoamericana sobre Calidad, Eficacia y Cambio en Educación, 8(1), 7-25.

Cervini, R. (2011). Equidad y oportunidad de aprender en la educación básica. Profesorado. Revista de Currículum y Formación de Profesorado, 15(3), 67-86.

Cervini, R., Dari, N. y Quiroz, S. (2010). Factores institucionales del logro en Matemática en la educación media de Argentina, 1998-2007: Una actualización. Revista Iberoamericana sobre Calidad, Eficacia y Cambio en Educación, 11(2), 121-148.

Contreras, D. (2001). Evaluating a voucher system in Chile. Individual, family and school characteristics (Documento de Trabajo No. 175, Facultad de Ciencias Económicas y Administrativas, Universidad de Chile).

Contreras, D., Bustos, S. y Sepulveda, P. (2010). When schools are the ones that choose: The effect of screening in Chile. Social Science Quarterly, 91(5), 1349-1368. doi:10.1111/j.15406237.2010.00735.x

Curi, A. y Menezes-Filho, N. A. (2009). A relação entre educação pré-primária, salários, escolaridade e proficiência escolar no Brasil. Estudos Econômicos, 39(4), 811-850. doi:10.1590/s0101-41612009000400005

Dirección Nacional de Información y Evaluación de la Calidad Educativa. (2007). Aspectos conceptuales y resultados nacionales - Operativo Nacional de Evaluación 2005. Buenos Aires: Autor.

Ferrer, G. (2006). Educational assessment systems in Latin America: Current practice and future challenge. Washington, DC: PREAL.

Franco, C., Ortigão, I., Albernaz, A., Bonamino, A., Aguiar, G., Alves, F. y Sátyro, N. (2007). Qualidade e equidade em educação: Reconsiderando o significado de "fatores intraescolares”. Ensaio: Avaliação e Políticas Públicas em Educação, 15(55), 277-298. doi:10.1590/s0 104-40362007000200007

Gonçalves, F. O. y França, M. T. A. (2008). Transmissão intergeracional de desigualdade e qualidade educacional: avaliando o sistema educacional brasileiro a partir do SAEB 2003. Ensaio: Avaliação e Políticas Públicas em Educação, 16(61), 639-662. doi:10.1590/010440362008000400009

Instituto Nacional de Estudos e Pesquisas Educacionais. (2007). SAEB - 2005: Primeiros Resultados: Médias de desempenho do SAEB/2005 em perspectiva comparada. Brasília: Autor.

Krawczyk, N. R. y Vieira, V. L. (2006). Homogeneity and heterogeneity on education systems in Argentine, Brazil, Chile and Mexico. Cadernos de Pesquisa, 36(129), 673-704. doi:10.1590/s0100-15742006000300009

Krawczyk, N. R. y Vieira, V. L. (2010). A reforma educacional na América Latina nos anos 90: Uma perspectiva histórico-sociológica. Revista Latinoamericana de Educación Comparada, $1(1), 10-17$.

Laros, J. A., Marciano, J. L. P. y Andrade, J. M. (2012). Fatores associados ao desempenho escolar em Português: Um estudo multinível por regiões. Ensaio: Avaliação e Políticas Públicas em Educação, 20, 623-646.

McEwan, P. J. (2003). Peer effects on student achievement: Evidence from Chile. Economics of Education Review, 22(2), 131-141. doi:10.1016/s0272-7757(02)00005-5

Meckes, L. (2003). SIMCE: Su desarrollo y sus desafíos actuales. Revista Pensamiento Educativo, $33(2), 160-178$. 
Mizala, A. y Romaguera, P. (2002). Equity and educational performance. Economía, 2(2), 219-273. doi:10.1353/eco.2002.0009

Mizala, A. y Torche, F. (2012). Bringing the schools back in: The stratification of educational achievement in the Chilean voucher system. International Journal of Educational Development, 32(1), 132-144. doi:10.1016/j.ijedudev.2010.09.004

Murillo, F. J. (2005). La investigación sobre eficacia escolar. Barcelona: Octaedro.

Murillo, F. J. (2007). School effectiveness research in Latin America. In T. Townsend (Ed.), International Handbook of School Effectiveness and Improvement (pp. 75-92). Nova York: Springer.

Newman M., Garrett Z., Elbourne D., Bradley S., Noden P., Taylor J. y West A. (2006). Does secondary school size make a difference? A systematic review. Education Research Review, 1(1), 41-60. doi:10.1016/j.edurev.2006.03.001

Oliveira, P. R., Belluzzo, W. y Pazello, E. T. (2013). The public-private test score gap in Brazil. Economics of Education Review, 35, 120-133. doi:10.1016/j.econedurev.2013.04.003

Paredes, V. (2014). A teacher like me or a student like me? Role model versus teacher bias effect. Economics of Education Review, 39, 38-49. doi:10.1016/j.econedurev.2013.12.001

Roemer, J. (1998). Equality of opportunity. Cambridge, MA: Harvard University Press.

Rodrigues, C. G., Rios-Neto, E. L. G. y Pinto, C. C. D. X. (2011). Diferenças intertemporais na média e distribuição do desempenho escolar no Brasil: O papel do nível socioeconômico, 1997-2005. Revista Brasileira de Estudos de População, 28(1), 5-36. doi:10.1590/s010230982011000100002

Soares, J. F. y Alves, M. T. G. (2003). Desigualdades raciais no sistema brasileiro de educação básica. Educação e Pesquisa, 29(1), 147-165. doi:10.1590/s 1517-97022003000100011

Soares, J. F. (2004). Qualidade e equidade na educação básica Brasileira: A evidência do SAEB2001. Archivos Analíticos de Políticas Educativas, 12(38), 1-28.

Soares, J. F. y Candian, J. (2007). O efeito da escola básica brasileira: As evidências do PISA e do SAEB. Revista Contemporânea de Educação, 2(4), 1-12.

Teodorovic, J. (2009). School Effectiveness: Literature review. Zbornik Instituta za pedagoska istrazivanja, 41(1), 7-24. doi:10.2298/zipio901007t.

Thieme, C., Prior, D. y Tortosa-Ausina, E. (2012). A multilevel decomposition of school performance using robust nonparametric frontier techniques. Economics of Education Review, 32, 104-121. doi:10.1016/j.econedurev.2012.08.002

Townsend, T. (2007). International Handbook of School Effectiveness and Improvement. Nueva York: Springer.

UNESCO (2015). Education for all 2000-2015: Achievements and challenges. UNESCO Publishing.

Wainer, J., Dwyer, T., Dutra, R. S., Covic, A., Magalhães, V. B., Ferreira, L. R. R., Pimenta, V. A. e Claudio, K. (2008). Too much computer and internet use is bad for you, especially if you are young and poor: Results from the 2001 Brazilian SAEB. Computers and Education, 51(4), 1417-1429. doi:10.1016/j.compedu.2007.12.007

Waltenberg, F. y Vandenberghe, V. (2007). What does it take to achieve equality of opportunity in education? An empirical investigation based on Brazilian data. Economics of Education Review, 26(6), 709-723. doi:10.1016/j.econedurev.2007.09.002 


\section{Breve CV de los autores}

\section{Luís Gustavo Amaral Vinha}

Prof. Luís Gustavo es Doctor en Psicología Social, Universidad de Brasília (UnB), Máster en Estadística, Universidad de São Paulo. Actualmente es profesor adjunto en el Departamento de Estadística de la UnB. Tiene experiencia en una amplia variedad de técnicas estadísticas como: control estadístico del proceso, métodos multivariados, análisis de factores, teoría de la respuesta al ítem, modelos jerárquicos e tratamiento de datos ausentes. Ha trabajado principalmente en las áreas de evaluación educativa a gran escala e evaluación de políticas y programas sociales.

\section{Jacob Arie Laros}

Prof. Jacob Arie Laros estudió Psicología en la Rijksuniversiteit de Groninga (Holanda). Obtuvo el título de PhD en Psicología en el año 1991. En 1995 fue contratado por el Instituto de Psicología de Universidad de Brasilia (UnB). Ocupó el cargo de coordinador del Programa de Apoyo a la Evaluación Educativa (PROAV - Brasilia) entre los años 1998 y 2001. Ese proyecto, que recibió apoyo de la CAPES, tenía como objetivo la divulgación del desarrollo de métodos y técnicas de evaluación educativa para apoyar las demandas de evaluación de los sistemas educacionales de gran escala del Brasil. Actualmente es profesor asociado IV en el Instituto de Psicología de la UnB. Es coordinador del laboratorio META (Métodos y Técnicas de Evaluación) y autor de dos test no-verbales de inteligencia: el SON-R para niños de 2,5 a 7 años y el SON-R para personas de 6 a 40 años. 